\title{
Spindle cell myoepithelioma of the parotid gland
}

\author{
Suk Joon $\mathrm{Oh}^{1}$, \\ Dukju Moon ${ }^{2}$ \\ Departments of ${ }^{1}$ Burn Reconstructive \\ Surgery and ${ }^{2}$ Burn Surgery, Bestian \\ Seoul Hospital, Seoul, Korea
}

\begin{abstract}
Myoepithelioma was recognized as a histological distinct entity by the World Health Organization (WHO) in 1991. Myoepithelial cells are believed to be of ectodermal origin. In salivary glands, the myoepithelial cells that surround the intercalated ducts are spindled, which is in contrast to the large stellate ones that envelop the acini. Myoepithelioma is a benign salivary gland tumor that consists entirely of myoepithelial cells. A 53-year-old man presented with a 1-year history of a painless mass originating from the right parotid gland. The mass grew rapidly reaching a size of approximately $6 \mathrm{~cm}$. The patient had no facial paralysis. The authors performed right parotidectomy. Immunohistochemistry study of this tumor showed that it was positive for vimentin, positive for S-100, focally positive for pancytokeratin, and focally positive for p63 and that it had a Ki-67 labeling index (below 10\%). Additionally, the tumor was negative for epithelial membrane antigen, negative for actin, negative for desmin, negative for CD34 and negative for anaplastic lymphoma kinase. The authors present a case of benign spindle cell myoepithelioma of the parotid gland in a 53-year-old man diagnosed after immunohistochemistry study, describing its importance, along with a brief review of the literature.
\end{abstract}

Keywords: Myoepithelioma / Parotid neoplasm

\section{INTRODUCTION}

As it is noted by Savera and Zarbo [1], myoepithelial cells of the salivary gland were first described by Zimmerman in 1898. In 1943 , Sheldon was the first to classify salivary gland tumors as myoepitheliomas in a review of 57 mixed tumors of the salivary glands, in which three salivary gland tumors were classified as myoepitheliomas. Myoepithelial cells are believed to be of an ectodermal origin. They envelop the glandular, acinar, and ductal elements of various organs, especially the breasts and salivary glands. In the salivary glands, the myoepithelial cells that surround the intercalated ducts are spindled, which is in contrast to the large satellite ones that envelop the acini [1].

Myoepithelioma was recognized as a histologically distinct entity by the World Health Organization (WHO) in 1991 [2]. It

Correspondence: Suk Joon Oh

Department of Burn Reconstructive Surgery, Bestian Seoul Hospital, 429 Dogok-ro, Gangnam-gu, Seoul 06208, Korea

E-mail: sjoh46@nate.com is a benign salivary gland tumor that consists entirely of myoepithelial cells with variable cellular morphologies, including spindle, epithelioid, plasmacytoid, and clear cells. A myoepithelioma can be composed of one or a mixture of these cell types, and a variable stromal component is seen in these tumors.

Myoepitheliomas are unusual and uncommon tumors that constitute only $1 \%$ to $1.5 \%$ of all salivary gland neoplasms $[3,4]$.

The pathologist and treating oncologist should be aware of this rare diagnosis, and it should always be a part of the differential diagnosis. However, only a morphological examination may be insufficient, even for an experienced pathologist in some cases. In such a case, the role of immunohistochemistry studies comes into play, and it should be considered to identify the diagnosis [5]. A 53-year-old male patient diagnosed with benign spindle cell myoepithelioma of the parotid gland after immunohistochemical study describes the importance of benign spindle cell myoepithelioma of the parotid gland in this literature review. 


\section{CASE REPORT}

A 53-year-old man presented with a 1-year history of a painless mass originating from the right parotid gland (Fig. 1). The mass grew rapidly, reaching a size of approximately $6 \mathrm{~cm}$. The patient had no facial paralysis. Ultrasonography revealed an ovoid, homogenous, exophytic and lobulated isoechoic right parotid

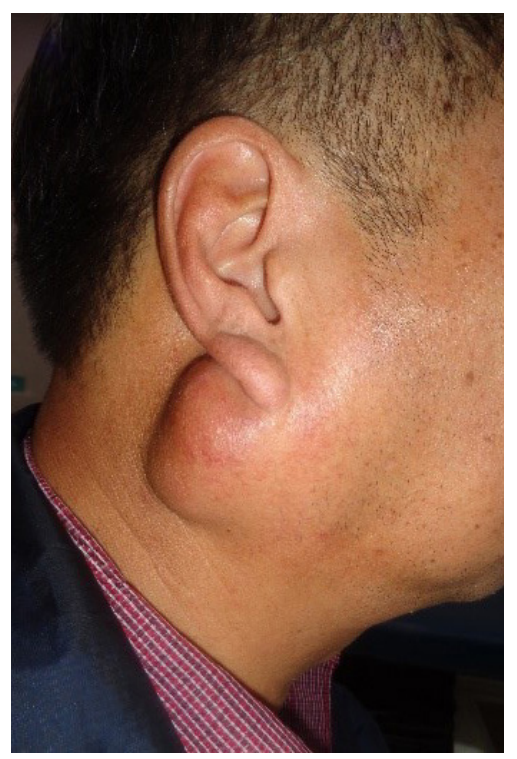

Fig. 1. A 53-year-old man presented with a 1-year history of a painless rapid growing mass in the region of the right parotid gland.
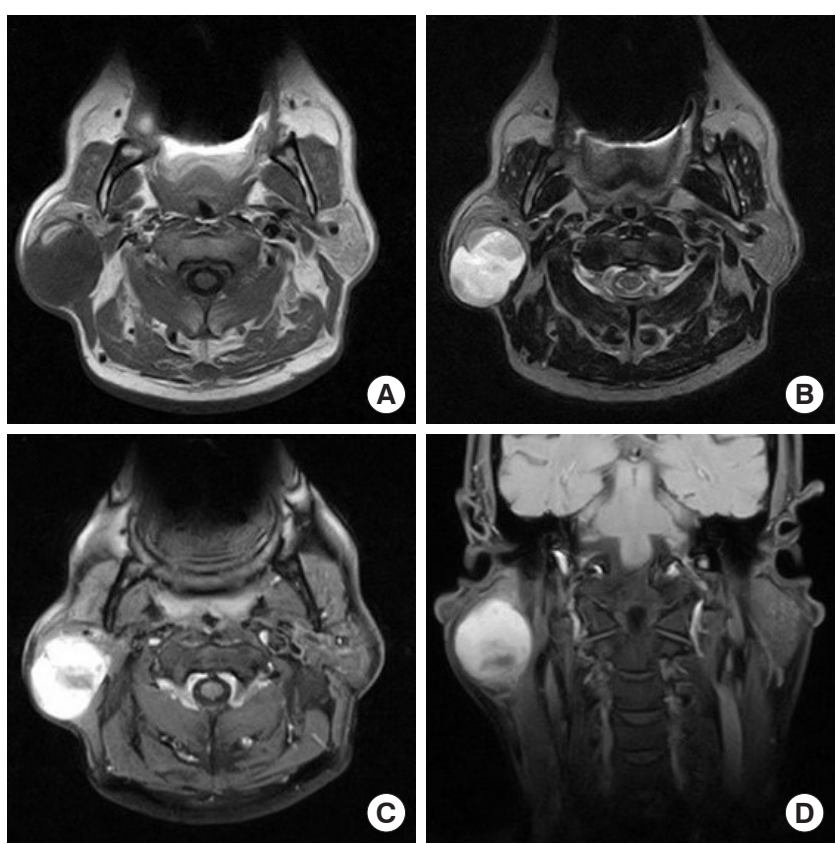

Fig. 2. Magnetic resonance images noted a large exophytic, wellmarginated and lobulated mass. T1W1 image (A), T2W1 image (B), T1W1-Gd-axial (C), and T1W1-Gd-coronal (D). T1WI, T1 weighted image; T2WI, T2 weighted image mass measuring $4.37 \times 3.92 \mathrm{~cm}$. Magnetic resonance images noted a large exophytic, lobulated mass. T1WI (T1 weighted image; Fig. 2A) and T2WI (T2 weighted image; Fig. 2B) as compared with T1WI-Gd (gadolinium) of the axial (Fig. 2C) and coronal (Fig. 2D) views showed more enhancement in the periphery than that in the center and a relatively well-marginated mass $(5.5 \times 4.37 \times 3.92 \mathrm{~cm})$ in the right parotid gland without enlarged cervical lymph nodes. Benign mixed tumor and mucoepidermoid carcinoma were suspected. Superficial parotid gland resection including two parotid and two jugular lymph nodes was performed.

The gross specimen received in formalin was a previously bisected salivary gland tumor surrounded by yellowish brown soft tissue, measuring $7 \times 6 \times 6 \mathrm{~cm}$ in total and weighing $68 \mathrm{~g}$. On sectioning, there was a well-encapsulated whitish yellow myxoid tumor with areas of hemorrhagic necrosis and focal cystic change. The tumor was $5.5 \times 4.5 \times 4.0 \mathrm{~cm}$ in size (Fig. 3).

The pathologist was initially suspicious of spindle cell myxoid tumor of an undetermined type with foci of an epithelial-like arrangement and hemorrhagic necrosis (Fig. 4a). Immunohistochemistry study showed that it was positive for vimentin (Fig. 4b), positive for S-100 (Fig. 4c), focally positive for pancytokeratin (Fig. 4d), and focally positive for p63 (Fig. 4e) and that it had a Ki-67 labeling index (below 10\%) (Fig. 4f). Additionally, the analysis showed that the tumor was negative for EMA, negative for actin, negative for desmin, negative for CD34 and negative for ALK (Table 1). Therefore, the pathologic diagnosis was benign spindle cell type myoepithelioma with necrosis. There was no evidence of disease at the 3-year follow-up.

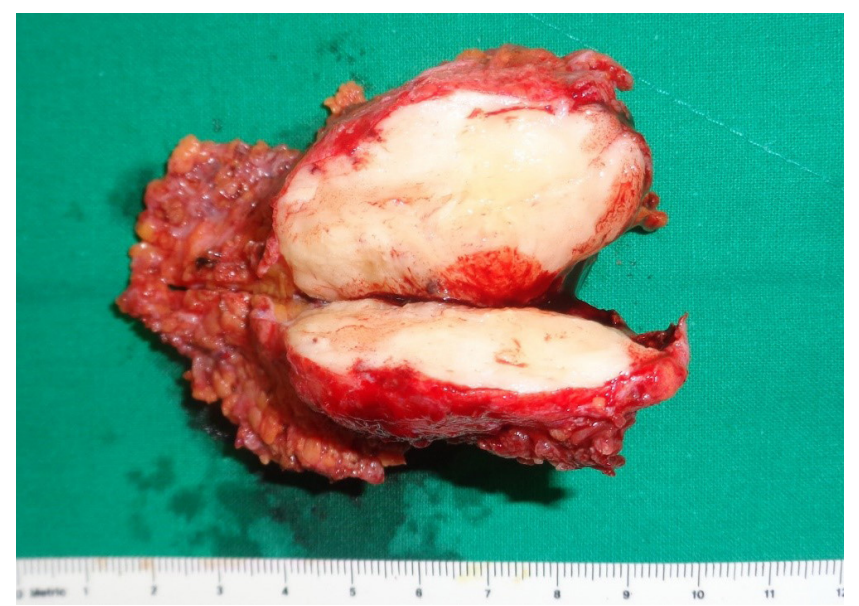

Fig. 3. The bisected specimen of the parotid mass consisted of an encapsulated, soft, lobulated mass with a yellow-to-white on-cut surface. 


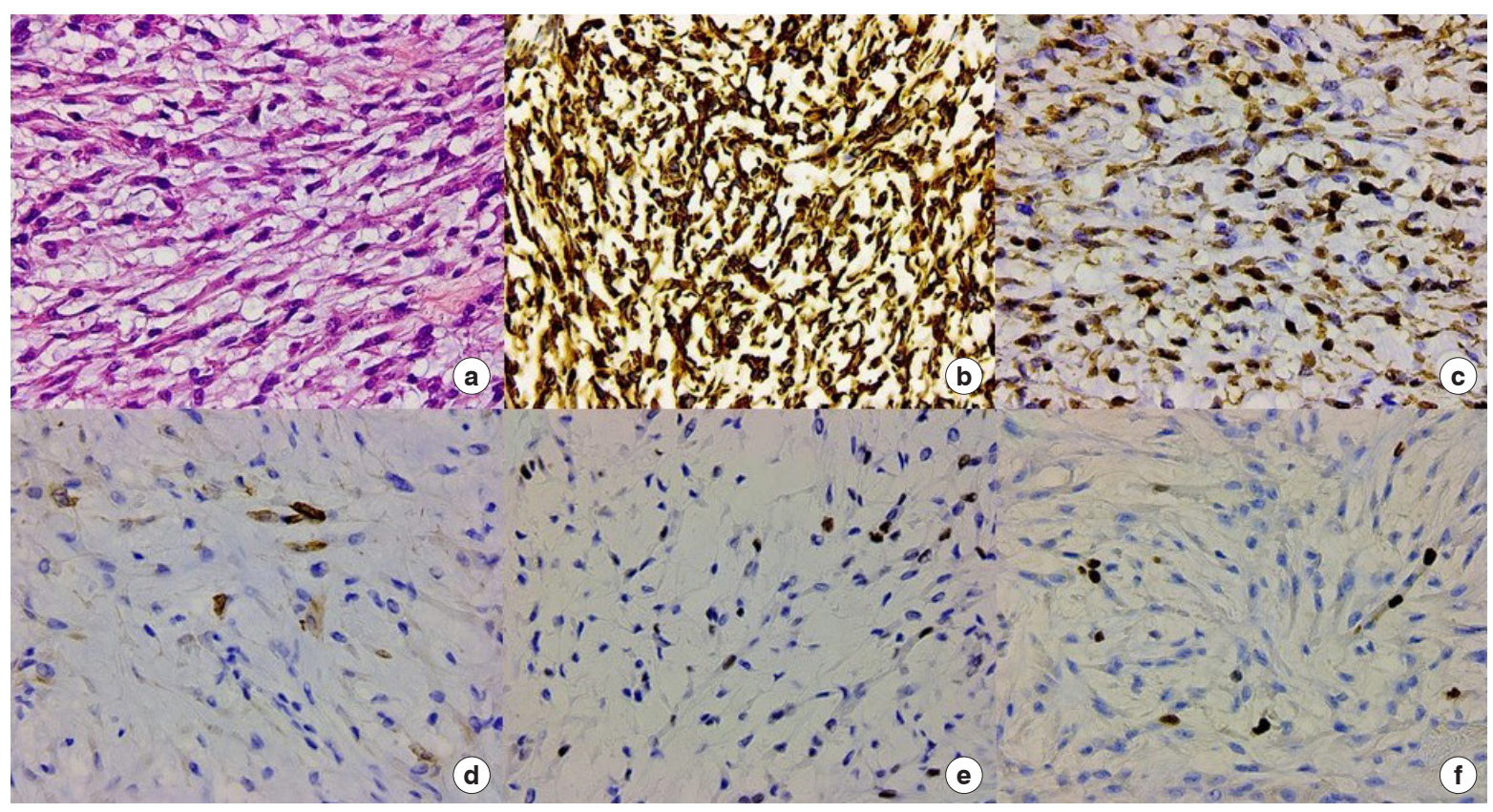

Fig. 4. Histological findings. (a) Histological study of the tumor showed spindle cell arrangement (H\&E, $\times 400)$. Immunohistochemistry study showed that it was positive for vimentin $(b, \times 400)$, positive for S-100 (c, $\times 400)$, focally positive for pancytokeratin $(d, \times 400)$, and focally positive for p63 (e, $\times 400)$ and that Ki-67 was below the $10 \%$ labeling index $(f, \times 400)$.

Table 1. Management record of immunohistochemistry

\begin{tabular}{|c|c|c|c|c|c|c|c|c|}
\hline No & Antigen & Company & Country & Dilution & Source & Clone & Test & Result \\
\hline 1 & Vimentin & Dako & Denmark & $1: 100$ & Mouse & V9 & Polymer (Omnis) & Positive \\
\hline 2 & S-100 & Dako & Denmark & $1: 2,000$ & Rabbit & Rabbit poly & Polymer (Omnis) & Positive \\
\hline 3 & Pancytokeratin (CKAE1/AE3) & Dako & Denmark & $1: 400$ & Mouse & AE1/AE3 & Polymer (Omnis) & Focally positive \\
\hline 4 & P63 & Dako & Denmark & $1: 200$ & Mouse & DAK-p63 & Polymer (Omnis) & Focally positive \\
\hline 5 & Ki-67 & Roche & USA & Ready to use & Mouse & MIB-1 & Multimer (Ventana) & Below $10 \%$ in hot spot \\
\hline 6 & EMA & Dako & Denmark & $1: 200$ & Mouse & E29 & Polymer (Omnis) & Negative \\
\hline 7 & Actin & NOVOCASTRA & UK & $1: 100$ & Mouse & asm-1 & Polymer (Omnis) & Negative \\
\hline 8 & Desmin & Dako & Denmark & $1: 50$ & Mouse & D33 & Polymer (Omnis) & Negative \\
\hline 9 & CD34 & Dako & Denmark & $1: 100$ & Mouse & QBEnd 10 & Polymer (Omnis) & Negative \\
\hline 10 & ALK-1(p80) & NOVOCASTRA & UK & $1: 50$ & Mouse & $5 \mathrm{~A} 4$ & Multimer (Ventana) & Negative \\
\hline
\end{tabular}

EMA, epithelial membrane antigen; ALK, anaplastic lymphoma kinase.

\section{DISCUSSION}

In salivary glands and other exocrine glands, there are starshaped cells of ectodermal origin lying between the basal lamina and the acinar and ductal cells. These cells structurally resemble epithelial cells and smooth muscles and are thus referred to as myoepithelial cells. The contraction of myoepithelial cells facilitates an expulsive secretion by rupturing "ripe" mucous cells, reducing the luminal volume and preventing the distention of acini, and other functions of myoepithelial cells are for the promotion of epithelial cell differentiation and sensory chemoreceptors, maintenance of gland patency, transportation of metabolites, formation and maintenance of the basement membrane and tumor suppression. Myoepithelial cells are potentially differentiated into various morphologic cell types, such as the angulate/basaloid, epithelioid, clear, spindle and plasmacytoid (hyaline) cell types [6]. The different architectural arrangements found in myoepitheliomatous zones of a tumor are somewhat dependent on the type and quantity of extracellular matrix production associated with cytological differentiation, which can be classified as follows: myxoid, solid (non-myxoid), reticular, microcystic/pseudocystic and cribriform/pseudoglandular patterns [1]. Myoepitheliomas are unusual and uncommon tumors that constitute only $11.5 \%$ of all salivary gland neoplasms [3,4]. An equal sex distribution is seen in most case series [3]. The average age of tumor incidence in affected indi- 
viduals is 44 years, with there being a wide age range, from 9 to 85 years [7]. The parotid gland is the primary site of occurrence of most reported myoepitheliomas (40\%-50\%), followed by the minor salivary glands as the second-most preferred site (of which the palate is the most common location [21\%]) $[3,5]$. The submandibular gland accounts for $10 \%$ of tumors.

Myoepitheliomas are well-circumscribed, gray-white/tan/yellow solid masses (average size, $3-5 \mathrm{~cm}$ ) with a smooth outline [3]. Degenerative changes are usually not a feature. Tumors arising in the parotid gland are usually encapsulated, in contrast to those arising in other major glands (submandibular and sublingual) or in the minor salivary glands, which tend to lack a capsule or only have a partial capsule but are invariably wellcircumscribed. Computed tomography imaging findings of benign myoepitheliomas were well-circumscribed, enhanced mass lesions with smooth or lobulated margins located chiefly in the superficial lobe and abutted on the capsule of the gland similar to that of low-grade malignant tumors [8].

Myoepithelial cells represent cell types such as epithelioid (most common), spindle, plasmacytoid, clear (least common) or mixed type cell types [4]. A mixture of these subtypes may be present in one tumor. Spindle cell myoepitheliomas have central fusiform/cigar-shaped nuclei, an eosinophilic cytoplasm, and tapered ends, and they are usually arranged in interlacing fascicles.

Myoepithelioma cells have been usually shown to be positive for cytokeratins (e.g., AE1/AE3, CK 5/6, Cam 5.2, CK-7, and CK-14) and vimentin markers in immunohistochemistry. Vimentin is reported to be positive in neoplastic myoepithelial cells and negative in normal myoepithelial cells. The neoplastic transformation of myoepithelial cells can result in a loss or modification of their smooth muscle phenotype [7]. S-100 is usually positive in neoplastic myoepithelial cells, but it is not in normal salivary gland myoepithelial cells. Calponin is the most sensitive myogenic marker $[9,10]$. Another variable of positivity is seen in smooth muscle actin, muscle-specific actin, smooth muscle myosin, p63 protein and glial fibrillary acidic protein markers. Many investigators have found that cells with a spindle cell morphology have the strongest immunoreactivity to smooth muscle markers, followed by epithelioid cells, with the plasmacytoid and clear cells showing a lesser, if not an absent, activity on occasion [11]. Neoplastic myoepithelium has been well established to not always retain actin expression $[9,12]$. Fortunately, many such confirmations can be resolved by using a combination of these markers. Usually, a combination of a keratin in conjunction with the detection of S-100, vimentin, and/or a myogenic marker is required for the confirmation of the diagnosis of myoepithelioma.
Myoepithelial carcinoma, which is known as malignant myoepithelioma, is the malignant counterpart of myoepithelioma. Malignant myoepithelioma has been added to the second edition of the WHO's histological classification of salivary gland tumors [2]. In regard to the differential diagnosis between benign and malignant myoepitheliomas, the results of Nagao et al. [10] were helpful in assessing the cell proliferative activity in those diagnosed with malignant myoepitheliomas with more than seven mitotic figures in 10 high-power fields or with a Ki67 level index of more than 10\%. According to Sciubba and Brannon [3], compared with pleomorphic adenomas, myoepitheliomas are less prone to recurrence after complete surgical resection, with only one recurrence in 16 cases observed over a period of 1 month to 7 years.

Treatment should be designed as for a benign salivary gland tumor with a margin of normal uninvolved tissue being included within the surgical excision. The recurrence rate is similar to that of pleomorphic adenomas (15\%-18\%) [13].

\section{NOTES}

\section{Conflict of interest}

No potential conflict of interest relevant to this article was reported.

\section{Ethical approval}

An IRB (2018-05-002) waiver for case reports was obtained from Bestian Seoul Hospital in Seoul, Republic of Korea. Written informed consent was obtained.

\section{Patient consent}

The patient provided written informed consent for the publication and the use of his images.

\section{ORCID}

Suk Joon Oh https://orcid.org/0000-0001-7793-6198

Dukju Moon https://orcid.org/0000-0002-0419-4936

\section{REFERENCES}

1. Savera AT, Zarbo RJ. Defining the role of myoepithelium in salivary gland neoplasia. Adv Anat Pathol 2004;11:69-85.

2. Seifert G, Sobin LH. Myoepithelioma. World Health Organization international histological classification of tumours: histological typing of salivary gland tumours. 2nd ed. Berlin: Springer-Verlag; 1991.

3. Sciubba JJ, Brannon RB. Myoepithelioma of salivary glands: report of 23 cases. Cancer 1982;49:562-72. 
4. Dardick I, Thomas MJ, van Nostrand AW. Myoepithelioma: new concepts of histology and classification: a light and electron microscopic study. Ultrastruct Pathol 1989;13:187-224.

5. Kapoor A, Rajput PS, Bagri PK, Beniwal S, Kumar V, Kumar HS. Myoepithelioma of parotid: a case report and review of literature. J Oral Res Rev 2014;6:53-6.

6. Shah AA, Mulla AF, Mayank M. Pathophysiology of myoepithelial cells in salivary glands. J Oral Maxillofac Pathol 2016;20: 480-90.

7. Dardick I, van Nostrand AW. Myoepithelial cells in salivary gland tumors: revisited. Head Neck Surg 1985;7:395-408.

8. Wang S, Shi H, Wang L, Yu Q. Myoepithelioma of the parotid gland: CT imaging findings. AJNR Am J Neuroradiol 2008;29: 1372-5.

9. Savera AT, Gown AM, Zarbo RJ. Immunolocalization of three novel smooth muscle-specific proteins in salivary gland pleo- morphic adenoma: assessment of the morphogenetic role of myoepithelium. Mod Pathol 1997;10:1093-100.

10. Nagao T, Sugano I, Ishida Y, Tajima Y, Matsuzaki O, Konno A, et al. Salivary gland malignant myoepithelioma: a clinicopathologic and immunohistochemical study of ten cases. Cancer 1998;83:1292-9.

11. Hornick JL, Fletcher CD. Myoepithelial tumors of soft tissue: a clinicopathologic and immunohistochemical study of 101 cases with evaluation of prognostic parameters. Am J Surg Pathol 2003;27:1183-96.

12. Dardick I. Myoepithelioma: definitions and diagnostic criteria. Ultrastruct Pathol 1995;19:335-45.

13. Politi M, Toro C, Zerman N, Mariuzzi L, Robiony M. Myoepithelioma of the parotid gland: case report and review of literature. Oral Oncology Extra 2005;41:104-8. 